\section{Forty-five countries join Gum Health Day}

'Healthy gums, beautiful smile' was the slogan for Gum Health Day 2019, held worldwide on 12 May to raise awareness about the growing threat of gum diseases on general health and wellbeing.

This year's European Federation of Periodontology (EFP) campaign was joined by a record 45 national scientific societies of periodontology from Europe, the Americas, Africa, Middle East, and the Asia-Pacific region.

Gum diseases are the most common medical condition in adults, affecting eight in ten people over 35 -years-old. They are the main reason for adult tooth loss, which causes chewing problems and affects nutrition, quality of life, and self-esteem. Gum diseases are also linked with increased risks of cardiovascular disease, diabetes, chronic kidney disease, rheumatoid arthritis, and other serious, chronic conditions.

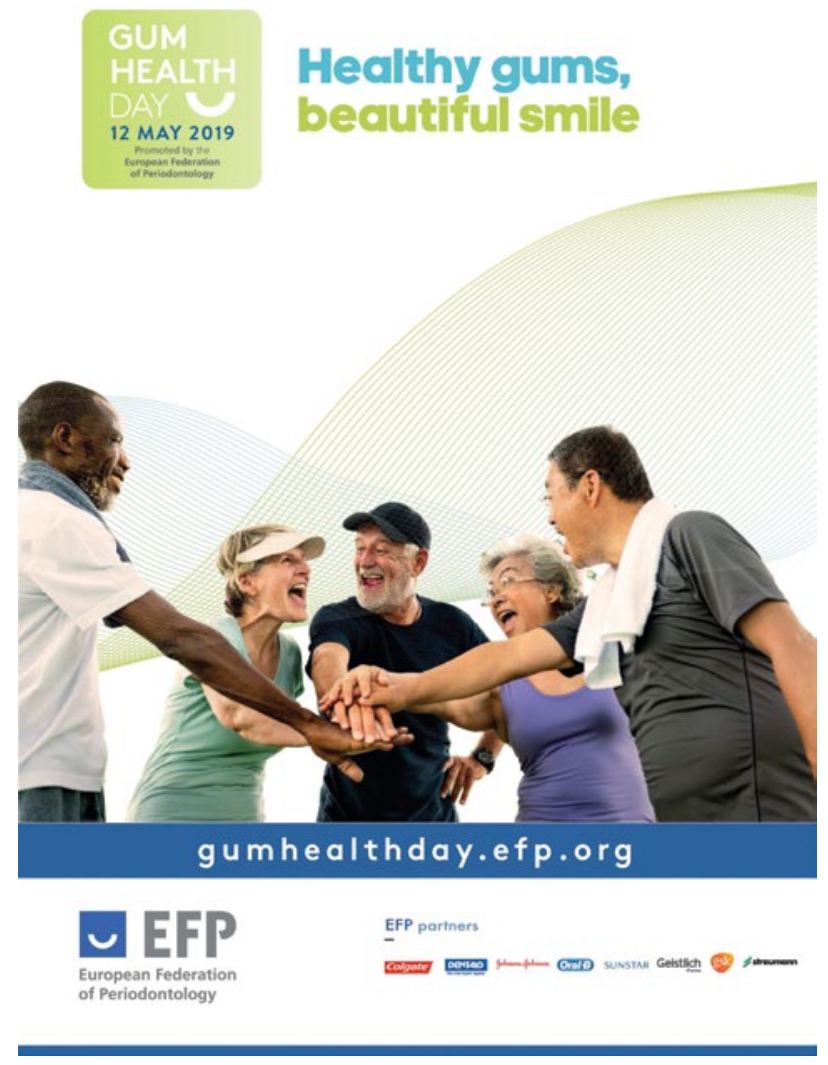

Professor Lior Shapira, EFP coordinator of Gum Health Day 2019, said: 'Looking after your gums is important for oral health and for overall health throughout life. Healthy gums also make beautiful smiles, as they preserve our teeth and facial contours.'

Activities were taking place in more than 100 cities with free periodontal screening, an app to assess your risk of gum disease, complimentary toothbrushes and toothpaste, lectures for dental students, workshops, videos, brochures, and media campaigns.

As part of Gum Health Day 2019, the EFP encourages dentists and other health professionals to sign and disseminate the EFP Manifesto Perio and General Health (http://www.efp.org/efp-manifesto/sign.php), an international call to action for the prevention, early detection and treatment of gum disease. By signing you will join the 1,000 individuals and organisations that have already endorsed it from around the world.

\section{BOOK REVIEW}

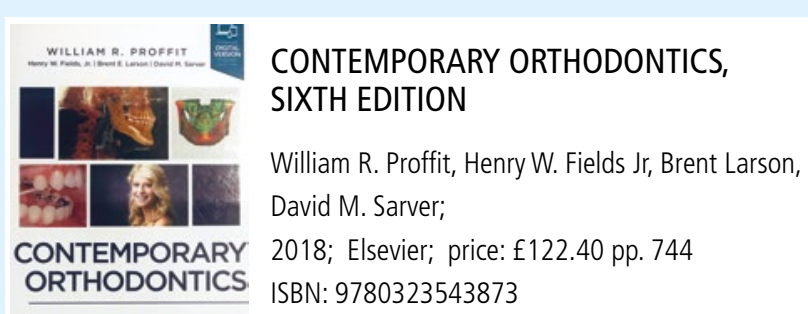

$\ldots$

Contemporary orthodontics has entered its sixth edition. It has been extensively revised by world-renowned orthodontic scholars led by William Proffit, with the main aim to provide an up to date overview of orthodontics accessible to all.

The new edition differs from previous versions as it comes with two additional chapters exploring they key topics of growth modification and multidimensional problems. It also includes a wealth of evidence-based case studies with great quality illustrations, which reinforces key concepts for clinical examinations and practice.

The book is divided into seven main sections, which are further subdivided into chapters covering succinct topics, totalling 20 chapters. Each section gives comprehensive coverage providing detailed information on orthodontic diagnosis, treatment planning and current treatment techniques. Additionally, each section has its own introduction and each chapter has its own outline, which is good as a quick reference.

The first section informs about the orthodontic problem such as the concepts of growth and development, and the aetiology of orthodontic problems. This follows nicely onto section two, which is all about orthodontic diagnosis and treatment planning. Sections three to six are based on orthodontic appliances, treatment in pre-adolescent children, growth modification and comprehensive orthodontic treatment in the early permanent dentition. The last section is dedicated to the treatment of adults.

The book is easy to navigate. It has a clear writing style and great organisation to help the reader comprehend challenging concepts. Coloured diagrams and illustrations are featured frequently throughout, emphasising key points well. The book initially provides a basic background of the topic in hand, which would be needed by dentists and useful for quick reference. It is then followed by more in depth information for orthodontic specialists. The book could benefit from learning objectives and the use of summaries.

The text is available as a textbook or ebook. The hardcover version also gives free electronic access to the text, including references linked to PubMed abstracts. This is a great advantage as it would be difficult to carry the book around daily due to it being quite heavy.

Overall, this new edition presents as an impressive holistic book, covering all key topics within the field of orthodontics in great detail. The book tailors to a wide audience, whether that be a study guide or a reference guide for dental students, practitioners and specialists. It is a valuable addition to anyone working, or aspiring to work, within orthodontics.

Rabia Dean 